\title{
Superconductivity in $\mathrm{LaRh}_{2} \mathrm{Ga}_{2}$ with
}

\section{non-centrosymmetric structure}

Yousuke Nakayama and Takahiro Muranaka*

Department of Engineering Science, the University of Electro-Communication, Chofu, Tokyo 182-8585, Japan

Address correspondence to E-mail: muranaka@uec.ac.jp

Table of Contents

Figure S1: EPMA analysis; (a) secondary-electron image, (b) compositional image and (c) topographical image of the sample

Figure S2: Rietveld refinement of multi-phase $\mathrm{LaRh}_{2} \mathrm{Ga}_{2}, \mathrm{LaRh}_{3}$ and $\mathrm{RhGa}_{3}$

Table S1: Reliability factors and mass fraction in Rietveld refinement of multi-phase

Table S2: Structural parameters of the $\mathrm{LaRh}_{2} \mathrm{Ga}_{2}$ phase

Figure S3: Temperature dependence of differential susceptibility $d \chi / d T$ during FC process 
Figure S1

Figures show various images of the sample, as follows: (a) secondary-electron image, (b) compositional image, and (c) topographical image of the sample. Electron probe microanalysis (EPMA) reveals that the matrix is the $\mathrm{LaRh}_{2} \mathrm{Ga}_{2}$ phase. The chemical composition was estimated to be $\mathrm{LaRh}_{2.02} \mathrm{Ga}_{2.35}$ accordingly the quantitative analysis of the typical grain.

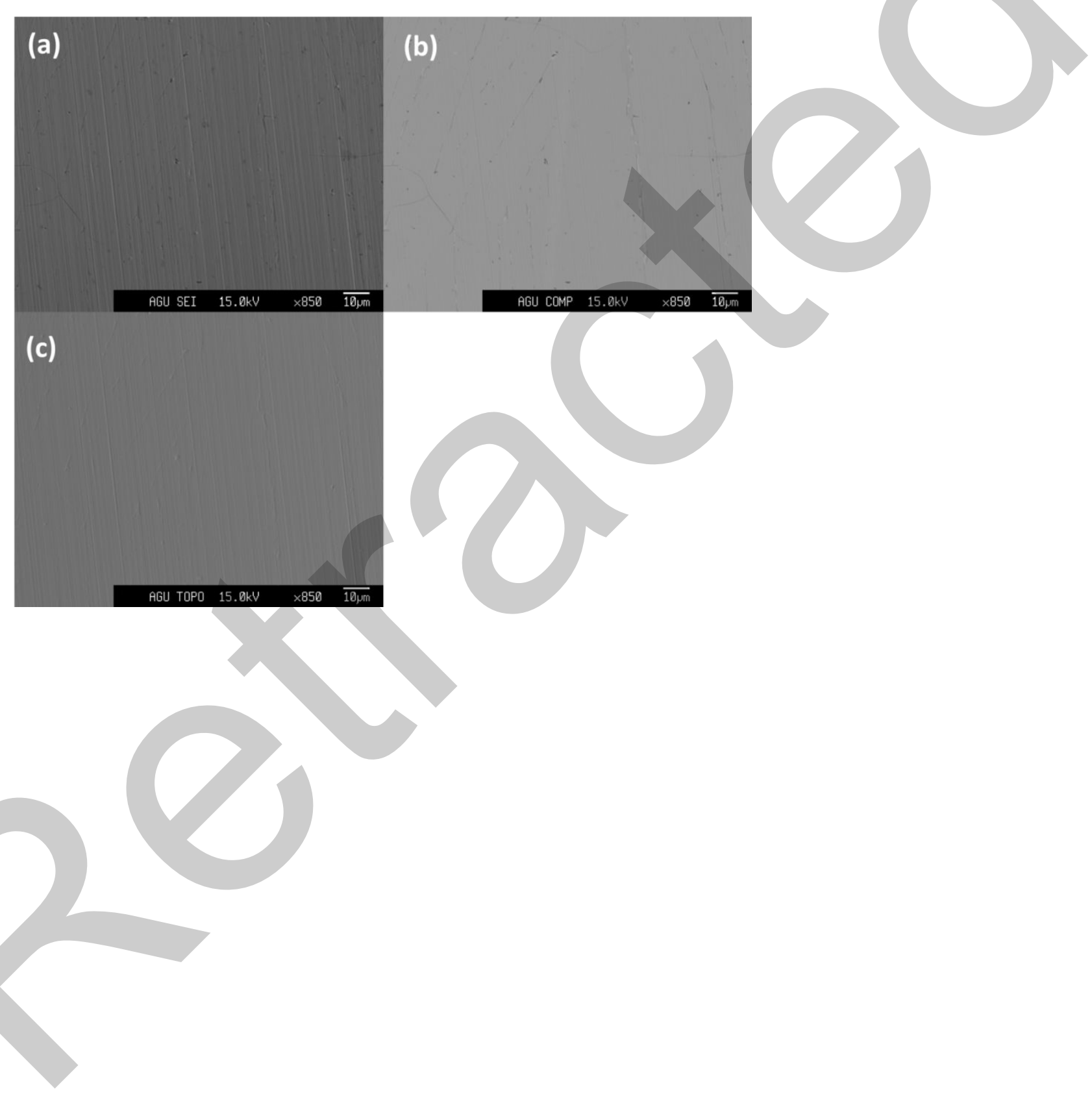


Figure S2

Rietveld refinement of multi-phase $\mathrm{LaRh}_{2} \mathrm{Ga}_{2}, \mathrm{LaRh}_{3}$ and $\mathrm{RhGa}_{3}$.

The red circles and black line correspond to the observed and calculated patterns, respectively. The blue line indicates the difference between the measured pattern and the calculated pattern, and the green lines denote the calculated peak positions for the $\mathrm{CaBe}_{2} \mathrm{Ge}_{2}$-type structure, $\mathrm{LaRh}_{3}$ and $\mathrm{RhGa}_{3}$.

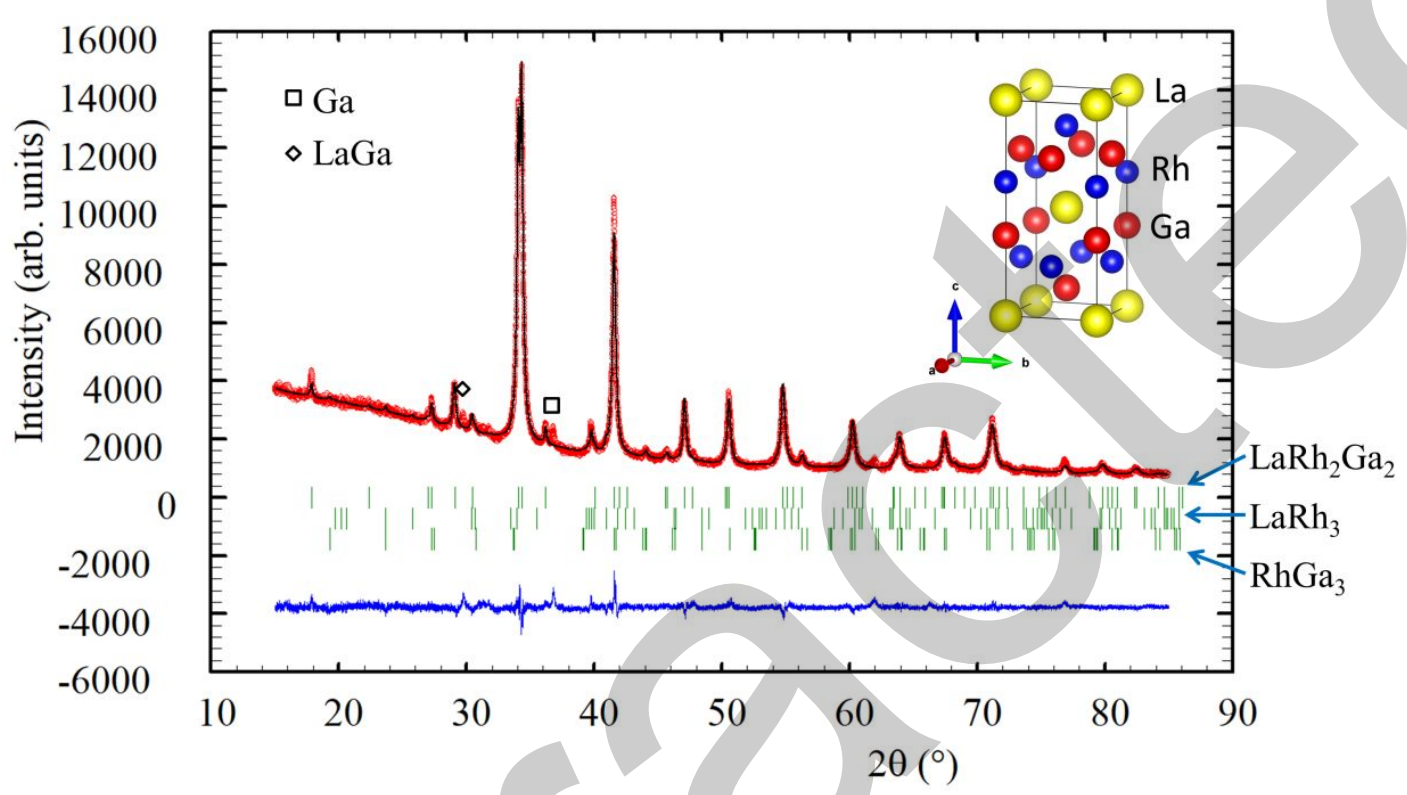


$\mathrm{S} 4$

Supporting Information for the paper

Table S1

Reliability factors and mass fraction in Rietveld refinement of multi-phase

\begin{tabular}{lcccc}
$R_{\mathrm{wp}}=3.943 \%$ & $R_{\mathrm{p}}=2.855 \%$ & $R_{\mathrm{R}}=15.502 \%$ & $R_{\mathrm{e}}=2.291 \%$ & $\mathrm{~S}=1.7213$ \\
& $R_{\mathrm{B}} \%$ & $R_{\mathrm{F}} \%$ & $R_{\mathrm{F}}^{2} \%$ & Mass fraction \% \\
\hline \hline $\mathrm{LaRh}_{2} \mathrm{Ga}_{2}$ & 1.787 & 1.114 & 1.005 & 92.04 \\
$\mathrm{LaRh}_{3}$ & 4.401 & 1.779 & 0.73 & 4.57 \\
$\mathrm{RhGa}_{3}$ & 12.665 & 2.746 & 1.246 & 3.40 \\
\hline
\end{tabular}

Table S2

Structural parameters of the $\mathrm{LaRh}_{2} \mathrm{Ga}_{2}$ phase

\begin{tabular}{ccccc} 
Atom & $\begin{array}{c}\text { Wyckoff } \\
\text { symbol }\end{array}$ & $x$ & $y$ & $z$ \\
\hline \hline $\mathrm{La}$ & $2 \mathrm{c}$ & $1 / 4$ & $1 / 4$ & $0.7418(2)$ \\
$\mathrm{Rh} 1$ & $2 \mathrm{c}$ & $1 / 4$ & $1 / 4$ & $0.1214(2)$ \\
$\mathrm{Rh} 2$ & $2 \mathrm{~b}$ & $3 / 4$ & $1 / 4$ & $1 / 2$ \\
$\mathrm{Ga} 1$ & $2 \mathrm{c}$ & $1 / 4$ & $1 / 4$ & $0.3704(4)$ \\
$\mathrm{Ga} 2$ & $2 \mathrm{a}$ & $3 / 4$ & $1 / 4$ & 0 \\
\hline
\end{tabular}

The space group is $P 4 / n m m . Z=2, a=4.3424(2) \AA$ and $c=9.9164(5) \AA$. The site occupancies were fixed at 1.0 
Supporting Information for the paper

Figure S3

Temperature dependence of the differential susceptibility $d \chi / d T$ during the FC process. The arrow indicates a change in the gradient.

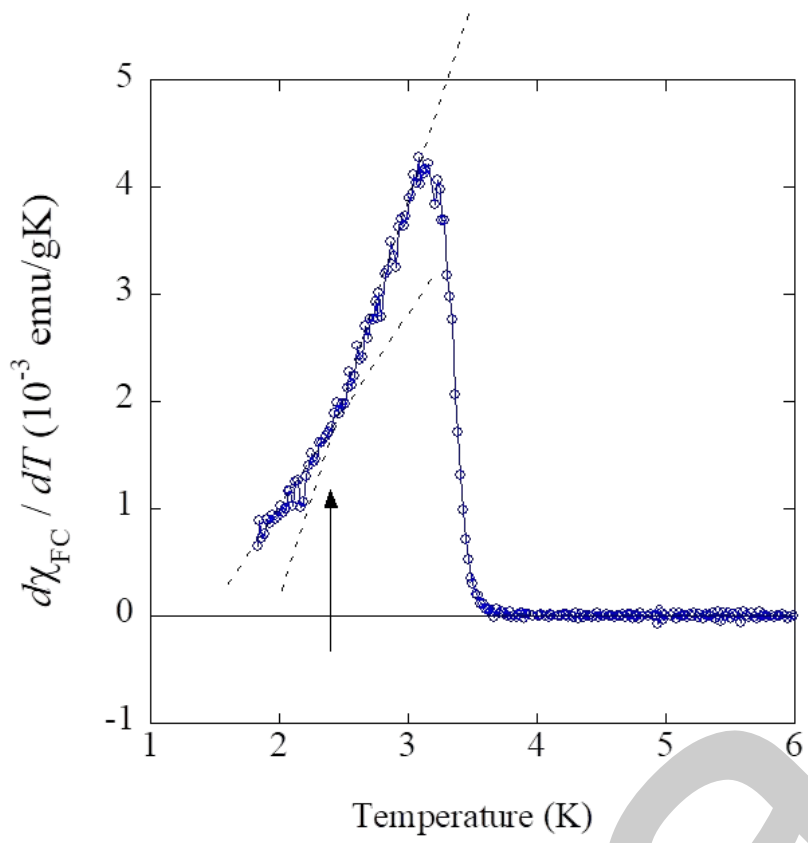

